Article

\title{
Design and Optimization of an Opened Suspended Core Fiber-Based SPR Sensor with Gold Cylinder Structures
}

\author{
Wentan Jiao ${ }^{1,+}{ }^{+}$Jinrong Liu ${ }^{2,+}$, Jing Zhang ${ }^{3}$, Guanjun Wang ${ }^{2,4, *}$ and Mengxing Huang ${ }^{4, *}$ \\ 1 College of Electrical Engineering and Automation, Luoyang Institute of Science and Technology, \\ Luoyang 471023, China; jwt@lit.edu.cn \\ 2 School of Information and Communication Engineering, North University of China, Taiyuan 030051, China; \\ liujinrong337@gmail.com \\ 3 School of Electronic and Information Engineering, Beihang University, Beijing 100083, China; \\ wentanjiao1982@163.com \\ 4 Collage of Information Science \& Technology, Hainan University, Haikou 570228, China \\ * Correspondence: wangguanjun@nuc.edu.cn (G.W.); huangmx09@hainu.edu.cn (M.H.) \\ + These authors contributed equally to this work.
}

Received: 22 February 2018; Accepted: 3 April 2018; Published: 9 April 2018

check for updates

\begin{abstract}
In this study, an opened three-hole suspended core fiber surface plasmon resonance sensor structure based on the combination of photonic crystal fiber and surface plasmon resonance (SPR) mechanism was proposed and analyzed. One hole in the clad layer was exposed to the outside, and a lay of gold cylinders with the same size and gap was placed along the inside of the opened hole. The existence of the gold cylinders could stimulate the SPR effect and selecting the suitable gaps between the cylinders could enhance the SPR effect and increase its sensitivity. Then COMSOL software was used to simulate how the cylinder diameter, the gaps between the cylinders, and the fluid refractive index variation affect the sensor's transmission loss spectrum, field enhancement effect, and the sensitivity. The optimized results show that the sensitivity of this proposed SPR sensor could be high, up to $1 \times 10^{-5} \mathrm{RIU} / \mathrm{nm}$, and it was much higher than the sensitivity of the existing photonic crystal fiber SPR sensor $\left(1 \times 10^{-4} \mathrm{RIU} / \mathrm{nm}\right)$, which was an order of magnitude improvement. This study could provide a reliable theoretical basis for future research and design of real-time and high-sensitivity opened fiber SPR sensors.
\end{abstract}

Keywords: suspended core fiber; surface plasmon resonance; opened fiber

\section{Introduction}

For conventional closed-hole micro-structured optical fiber for fluid testing, it takes a long time to fill the micro-holes with fluid to be measured. This slow filling rate greatly limits the application of conventional micro-structured optical fibers in fast fluidic detection [1-3]. The introduction of micro-structured optical fiber with side-opening structure (referred to as opened fiber) greatly reduces the huge contradiction between the slow fluid-filling rate of the traditional microstructured optical fiber fluidic sensing device and the requirement of fast fluidic detection. At the same time, it broadens the application prospect of microstructured optical fiber in the field of fast fluidic sensing [4-12]. Meanwhile, the opened microstructured optical fiber is also expected to enhance the degree of overlap and the interaction effect between the measured fluid and the optical field within the sensing region, thereby enhancing the sensing performance while achieving rapid detection at the same time $[4,5]$. In addition, by optimizing the optical fiber structure, sensing mechanism and signal demodulation methods [6], opened microstructured optical fiber fluidic sensing technology is expected to achieve 
more sensitive and flexible sensing features. In addition, it enables multi-parameters sensing of fluid properties. Assisted with the labeled biological antibodies or fluorescent substances and other measures [6,7], the applications of the proposed opened microstructured fiber can be broadened to biomolecular detection. The combination of opened microstructured optical fiber fluid sensing technology and microfluidic chip technology [8,9] will help improve the integration, portability and sensitivity of microfluidic chip detection systems and the fluid dynamic control of fiber fluidic sensing systems. In addition, the sensing mechanism of surface plasmon resonance (SPR) [10] and fiber grating [11] can further increase the sensitivity and extend the range of application of fluidic sensing.

By coating the metal film or the metal particle on the inner surface of an opened optical fiber micro-holes, the surface plasmon resonance (SPR) and the field enhancement can be effectively excited. Based on the upper phenomenon, an opened fiber SPR sensor with extremely high detection sensitivity can be formed. Compared with the prism-based SPR sensor, opened fiber SPR sensor has the advantages of small size, high sensitivity, and low cost. In addition, it is easy to implement the integrated and portable fluidic detection. The types of existing opened fiber used in SPR testing today cover tapered optical fibers [12], D-type optical fibers [13], PBG micro-structured optical fibers [14] and total reflection-type microstructured optical fibers [15]. The sensitivity of the optical fiber SPR sensor was closely related to the factors including the characteristics of the metal, the thickness of the coating and the refractive index range of the detection substance. By optimizing the above parameters, the sensor's sensitivity can be effectively improved.

Based on the opened suspended optical fiber structure, a novel optical fiber surface plasmon resonance sensor was designed here. It was characterized in that an air hole in the suspended core optical fiber is opened to the outside, and gold cylinders with the same size and same spacing were arranged along the axial direction of the fiber core inside the air hole cladding. The presence of gold cylinder can stimulate the SPR effect. At the same time, in different spectral bands, this study analyzes the impact of the diameter of the gold cylinder, the interval between the gold cylinder and the refractive index of the fluid under test on the sensitivity. The results show that by optimizing the parameters such as the diameter and spacing of the gold cylinder, its sensitivity $S$ could be greatly improved. The sensitivity of the optimized open-suspended optical fiber SPR sensor can be as high as $1 \times 10^{-5} \mathrm{RIU} / \mathrm{nm}$, which is an order of magnitude greater than the sensitivity of existing photonic crystal fiber SPR sensors $1 \times 10^{-4} \mathrm{RIU} / \mathrm{nm}[16]$. The content of this paper can provide useful reference for the design of real-time photonic crystal fiber SPR sensor in the future.

\section{Modeling}

Figure 1 is the cross-sectional schematic diagram of the opened three-hole suspension SPR sensor designed in this paper. There are three macropores in the suspension core fiber. One of the macropores is an open design for filling the fluid to be measured. The remaining two macropores are sealed and filled with air. The open pores are arranged with nanometer gold cylinders of the same size and pitch along the axial direction of the core. In practice, the above structure can be realized by self-assembly or nanoimprint method. The diameter of the gold cylinder is $d$, the distance between the gold cylinders is dgap, and the refractive index is denoted by nd. In the modeling process with COMSOL, we choose the core curvature $r=4 \mu \mathrm{m}$, cantilever thickness $c=0.4 \mu \mathrm{m}$, under this condition the structure can be maintained single-mode conditions [8]. The suspended optical fiber-based material is silica, whose refractive index $n_{c}$ under different wavelength conditions could be obtained by the Sellmeier equation [17]. Gold cylinder refractive index $n_{d}$ parameters can be obtained by reference to the relevant literature [18]. To implement the opened suspended core fiber-based SPR sensors for analytes, the opened holes could be coated with the gold nano cylinder using high pressure chemical vapor deposition (CVD) technique, wet chemistry deposition technique or nanoskiving [19-23], and then filled with the analytes. 


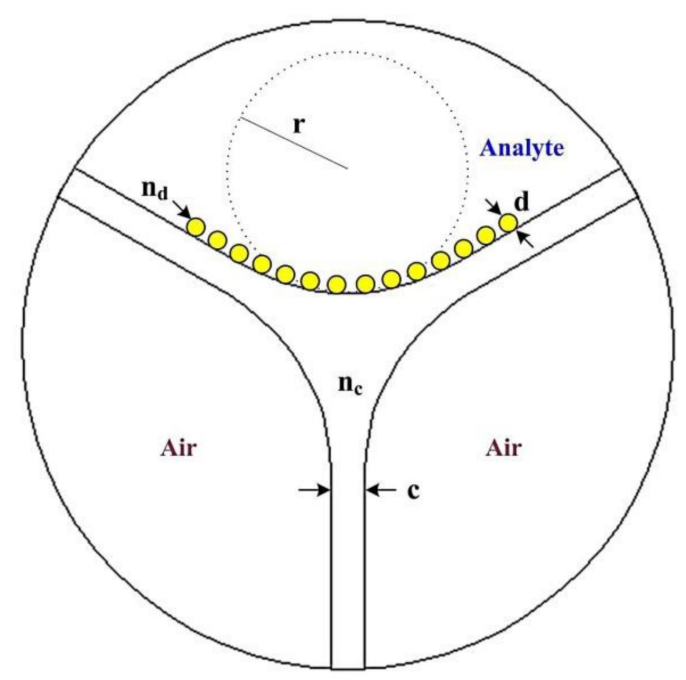

Figure 1. Schematic cross-section of the proposed fiber SPR sensor.

In microstructured optical fiber sensors, the mode power loss propagating forward along the $z$-axis can be expressed by Equation (1):

$$
P=P_{0} \mathrm{e}^{-\alpha z}
$$

where $P_{0}$ is the power at plane $z=0$. The mode attenuation constant is proportional to the imaginary part of the effective refractive index of the mode:

$$
\alpha=2 k_{0} \operatorname{Im}\left(n_{e f f}\right)
$$

Therefore, the attenuation coefficient can be used to quantify and evaluate Gaussian guided mode transmission loss.

In addition, the sensitivity of the SPR sensor can be expressed as:

$$
S=(\Delta \alpha / \Delta n) / \alpha_{a}
$$

where $\Delta n$ represents the offset value of the measured liquid refractive index value from $n_{a}=a$, $\Delta a$ represents the change value of the transmission loss for the wavelength when the liquid refractive index changes, $a_{a}$ represents the measured liquid refractive index $n_{a}=a$ fiber transmission loss spectrum.

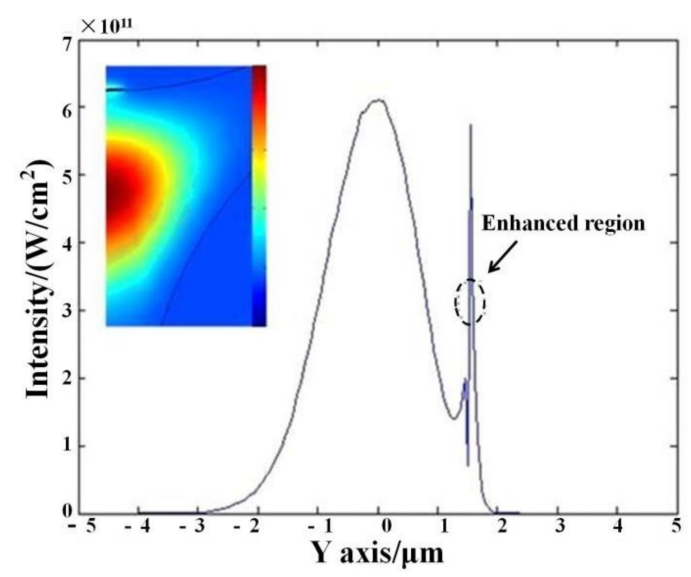

Figure 2. Spatial distribution of the sensor pattern. 
Figure 2 analyzes the spatial energy distribution of the proposed sensor pattern (gold cylinder diameter $d=35 \mathrm{~nm}$, transmission wavelength $l=530 \mathrm{~nm}$ ). The gap of the gold cylinders is set as $0.5 \mathrm{~nm}$, while for the entire loss peak, the energy distribution in the sensor-mode cantilever region is very close to the Gaussian type. The gold cylinder region has a strong energy distribution (field enhancement), the gold cylinder also formed a direct resonance effect. The mode field amplitude locally attenuates at the interface between the gold cylinder and the liquid being measured, which is characteristic of the surface plasmon resonance effect. Here the color/unit definition of the intensity bar is consistent with the intensity of $\mathrm{Y}$ axis, as it originates from the Multiphysics COMSOL software (COMSOL, Inc., Burlington, MA, USA) and is a relative magnitude.

\section{Results and Analysis}

Based on the above introduction, the paper analyzes the field enhancement effect, the transmission loss spectrum and the relationship between the diameter and spacing of the gold cylinder, and obtains the optimal structural parameters of the SPR sensor finally. Then the sensitivity of these structures is analyzed to find the optimal structural parameters.

Firstly, we set the distance between the gold cylinder constant $1 \mathrm{~nm}$, set the measured liquid refractive index $n_{a}=1.333$, gradually changing the diameter $d$ of the gold cylinder in the range of $30 \sim 65 \mathrm{~nm}$. At the same time, the relationship between the field enhancement of the fundamental mode and the diameter $d$ of the gold cylinder was measured, as shown in Figure 3. Here, the transmission wavelengths are the resonance peaks for $d=30-65 \mathrm{~nm}$, which are $516 \mathrm{~nm}, 530 \mathrm{~nm}, 545 \mathrm{~nm}, 561 \mathrm{~nm}, 614 \mathrm{~nm}$, $635 \mathrm{~nm}, 646 \mathrm{~nm}$ and $668 \mathrm{~nm}$ respectively; such data are consistent with the peak wavelengths in Figure 4 . The definition also originates from the Multiphysics COMSOL software and is just a relative magnitude. As the diameter of the gold cylinder increases from $30 \mathrm{~nm}$ to $55 \mathrm{~nm}$, the field enhancement effect of the fundamental mode increases gradually at the interface between the gold cylinder and the fluid under test. Correspondingly, in the area where the gold cylinder exists, the mode field amplitude decreases locally in both the core and the measured fluid. However, when the diameter of the gold cylinder is larger than $55 \mathrm{~nm}$, i.e., $d=55-65 \mathrm{~nm}$, the field enhancement effect of the fundamental mode diminishes as the diameter of the gold cylinder increases, and the local attenuation of the mode field amplitude in both materials is slightly enhanced. Compared with other diameters, the mode field amplitude decreased more in both the core and the measured fluid for $d=55-65 \mathrm{~nm}$. We suppose the reason is that with the increase, the transmission loss and the coupling loss between different nano cylinders also increase. Therefore, there is an optimal value that can make the field enhancement effect the most significant and maximize the effect of the plasma wave and the measured fluid. Here, $d=55 \mathrm{~nm}$ is a critical point and is also the size of the gold cylinder with the most significant field enhancement effect.

Secondly, we set the distance between the gold cylinder constant $0.5 \mathrm{~nm}$, set the liquid refractive index $n_{a}=1.333$ unchanged, gradually changing the diameter $d$ of the gold cylinder in the range of 30 $65 \mathrm{~nm}$. Meanwhile, the relationship between the fundamental mode transmission loss spectrum and the diameter $d$ of the gold cylinder was observed. As shown in Figure 4, with increase of the gold cylinder diameter $d$, the peak wavelength of mode loss peak changes too. It can be seen from Figure 4 that the wavelength of the plasma resonance peak shifts to a longer wavelength as the diameter of the gold cylinder increases over the wavelength range of $500 \mathrm{~nm}$ to $700 \mathrm{~nm}$. However, there is not a good linear relationship between the loss value at the resonance peak and the diameter of the gold cylinder. For example, with the gold cylinder diameter $d=30 \sim 55 \mathrm{~nm}$, as the diameter of the gold cylinder increases, the loss value at the resonance peak gradually increases and has the maximum value at $d=55 \mathrm{~nm}$. However, in the range of $d=55 \sim 65 \mathrm{~nm}$, with the increase of the diameter of the gold cylinder, the loss value at the resonance peak gradually decreases and the splitting peak appears. The existence of these splitting peaks is due to the mutual coupling of the plasmon waves between the gold cylinders, which needs to be considered in practical application. Here, the mode of maximum transmission loss is analyzed, as we think it is related with the maximum surface intensity and RI sensitivity. Of course, in actual application, it may be difficult to distinguish such modes. 


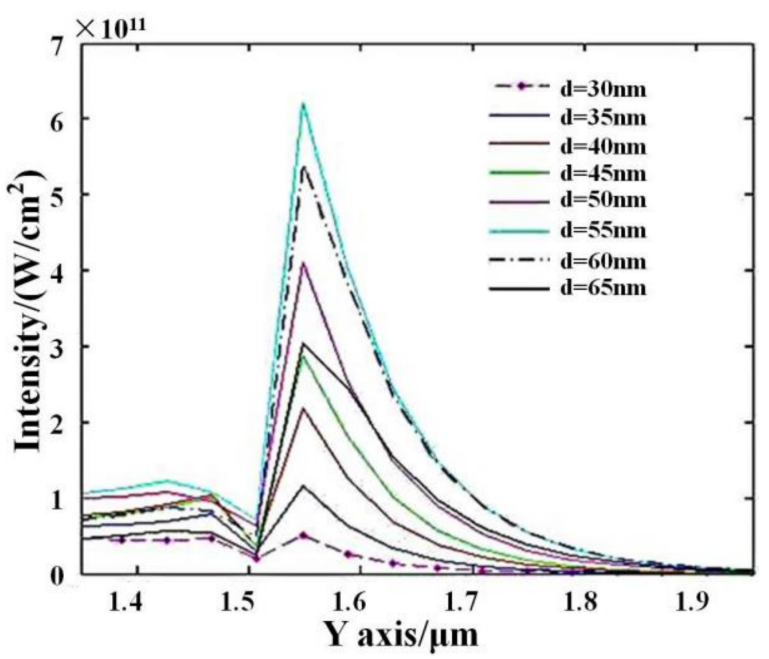

Figure 3. Relationship between the field enhancement of the fundamental mode and the diameter of the gold cylinder.

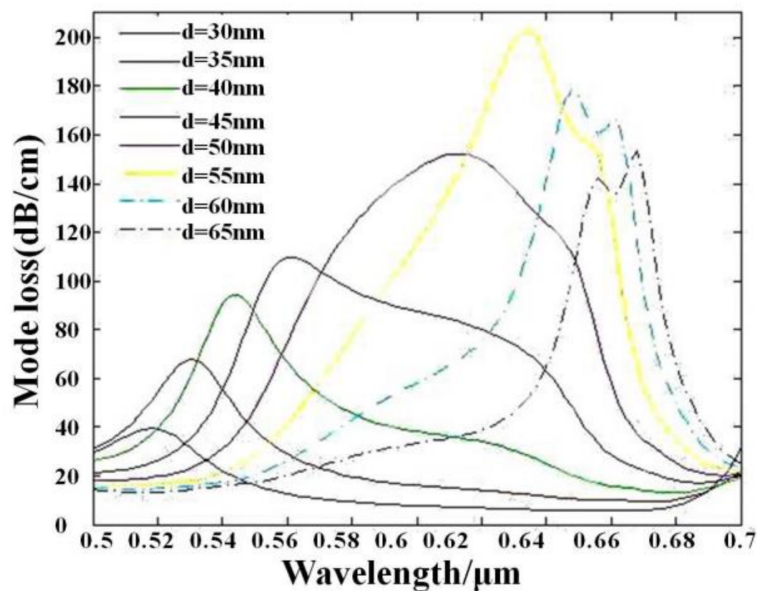

Figure 4. Relationship between transmission loss spectrum and gold cylinder diameter.

Based on the optimal value of the diameter of the gold cylinder $d=55 \mathrm{~nm}$, we study the optimal gap parameter of the gold cylinder. We set the gold cylinder diameter $d=55 \mathrm{~nm}$, gradually changing the gap between the gold cylinder in the range of $0.5 \sim 30 \mathrm{~nm}$, while the measured liquid refractive index is $n_{a}=1.333$. As shown in Figure 5, with the gap increases, the mode loss changes with wavelength. It can be seen from Figure 5 that as the gap increases in the range of $0.5-30 \mathrm{~nm}$, the resonant wavelength shifts to the longer wavelength. However, the peak loss at the resonant wavelength first increases significantly and then decreases, obtaining the maximum at gap $=20 \mathrm{~nm}$.

Therefore, the gold cylinder spacing theory is not the bigger the better, but instead there is an optimal value, making the formation of a resonant cavity between the gold cylinder. The plasmon resonate with each other in the resonator to enhance the SPR effect, so that the energy of the incident light can be transformed into plasma energy to a large extent. This shows that the effect on the transmission loss spectrum is that the resonance peak is the sharpest.

Based on the above analysis and simulation, this study is to choose the diameter of the gold cylinder and the corresponding gap, to optimize the sensitivity of the proposed fiber SPR sensor. As shown in Figure 6, the sensitivity of the three sensor structures was analyzed. The first structure was $d=45 \mathrm{~nm}$, gap $=20 \mathrm{~nm}$, the second structure was $d=50 \mathrm{~nm}, g a p=20 \mathrm{~nm}$ and the third structure was $d=55 \mathrm{~nm}$, gap $=20 \mathrm{~nm}$, assuming that the receiving end can detect the change of $1 \%$ of the transmission power. According to the sensitivity calculation formula $S=(\Delta \alpha / \Delta n) / \alpha_{a}$, the sensitivity of the first 
structure was S 94, that was, the change of the liquid refractive index in the order of $10^{-4} \mathrm{RIU} / \mathrm{nm}$ can be detected. the sensitivity of the second structure was S 189, that was, the change of the liquid refractive index in the order of $10^{-5} \mathrm{RIU} / \mathrm{nm}$ can be detected. Meanwhile, the sensitivity of the third structure was S 178, that is, the change of the liquid refractive index in the order of $10^{-5} \mathrm{RIU} / \mathrm{nm}$ can be detected. At the same time, it could be found that although the sensitivity of the first structure was relatively low, the sensitivity can maintain a high level in a long wavelength band. The second and third sensor structures have higher sensitivity, but the maximum sensitivity can only be maintained in a smaller band. Therefore, in practical applications, the working method can be selected reasonably to obtain the highest sensitivity or better sensitivity in a longer wavelength band.

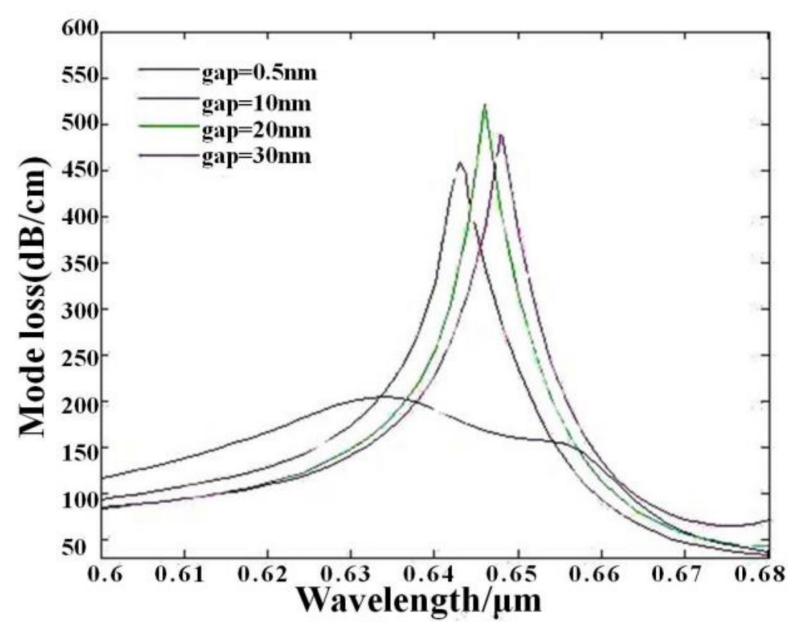

Figure 5. Relationship between transmission loss spectrum and gold cylinder spacing.

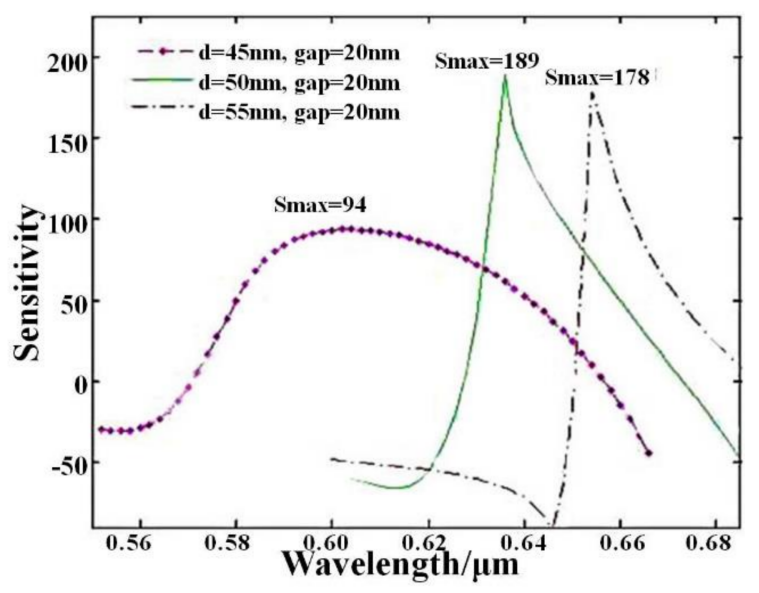

Figure 6. Further optimized structural sensitivity characteristics.

The region of refractive index used for the calculation is 1.333, which is close to the RI of water. Considering the detected RI value are near the index of water in most label-free sensing applications, the influence of RI was neglected. At the same time, in actual application, the sensor's structural parameters can be flexibly adjusted to meet different work requirements. For example, we can choose a long wavelength range of work or high sensitivity mode of operation.

\section{Conclusions}

In this paper, a novel open three-hole fiber SPR sensor structure with three-hole suspended core configuration was proposed. The influences of the diameter of the gold cylinder, the distance between the gold cylinders, and the refractive index of the fluid under test on the sensitivity were analyzed 
respectively by simulation. The results confirm that the sensitivity of the sensor can be improved by flexibly adjusting the diameter and spacing of the gold cylinder. We think, the optimized RI sensitivity mainly depends on the size and distribution of Au cylinder array, which could enhance the surface intensity and coupling effects between different Au cylinders under the optimized Au cylinder parameter. The effect of suspended core fiber is exciting and coupling the plasmon modes, which is of sub-importance. The simulation results show that the sensitivity of the optimized open-fiber optical fiber SPR sensor can be up to $1 \times 10^{-5} \mathrm{RIU} / \mathrm{nm}$, which is an order of magnitude greater than the sensitivity of existing photonic crystal fiber SPR sensors $\left(1 \times 10^{-4} \mathrm{RIU} / \mathrm{nm}, \mathrm{S}=70-80\right)$. The content of this study could provide useful reference for the design of photonic crystal fiber SPR sensor in the future.

Acknowledgments: This work was financially supported by National Key R\&D Program (2016YFC0101603), National Natural Science Foundation of China (grant no. 61405127), Program for the Outstanding Innovative Teams of Higher Learning Institutions of Shanxi, Scientific Technological Innovation Programs of Higher Education Institutions in Shanxi and Research start-up fund of Hainan University.

Author Contributions: Wentan Jiao, Jing Zhang and Guanjun Wang conceived, designed and performed the simulations; Jinrong Liu analyzed the data; Mengxing Huang contributed analysis tools; Jinrongliu, Guanjun Wang and Mengxing Huang wrote the paper.

Conflicts of Interest: The authors declare no conflict of interest.

\section{References}

1. Afshar, S.; Warren-Smith, S.C.; Monro, T.M. Enhancement of fluorescence-based sensing using microstructured optical fibres. Opt. Express 2007, 15, 17891-17901. [CrossRef] [PubMed]

2. Hoo, Y.L.; Jin, W.; Shi, C.; Ho, H.L.; Wang, D.N.; Ruan, S.C. Design and modeling of photonic crystal fiber gas sensor. Appl. Opt. 2003, 42, 3509-3515. [CrossRef] [PubMed]

3. Jensen, J.B.; Pedersen, L.H.; Hoiby, P.E.; Nielsen, L.B.; Hansen, T.P.; Folkenberg, J.R.; Riishede, J.; Noordegraaf, D.; Nielsen, K.; Carlsen, A.; et al. Photonic crystal fiber based evanescent-wave sensor for detection of biomolecules in aqueous solutions. Opt. Lett. 2004, 29, 1974-1976. [CrossRef] [PubMed]

4. Cox, F.M.; Lwin, R.; Large, M.C.J.; Cordeiro, C.M.B. Opening up optical fibers. Opt. Express 2007, 15, 11843-11848. [CrossRef] [PubMed]

5. Large, M.; Blacket, D.; Bunge, C.-A. Microstructured Polymer Optical Fibers Compared to Conventional POF: Novel Properties and Applications. IEEE J. Sens. 2010, 10, 1213-1217. [CrossRef]

6. Warren-Smith, S.C.; Ebendorff-Heidepriem, H.; Foo, T.C.; Moore, R.; Davis, C.; Monro, T.M. Exposed-core microstructured optical fibers for real-time fluorescence sensing. Opt. Express 2009, 17, 18533-18542. [CrossRef] [PubMed]

7. Weirich, J.; Lægsgaard, J.; Scolari, L.; Wei, L.; Alkeskjold, T.T.; Bjarklev, A. Biased liquid crystal infiltrated photonic bandgap fiber. Opt. Express 2009, 17, 4442-4453. [CrossRef]

8. Wang, G.; Wang, C.; Liu, S.; Zhao, J.; Liao, C.; Xu, X.; Liang, H.; Yin, G.; Wang, Y. Side-Opened Suspended Core Fiber-Based Surface Plasmon Resonance Sensor. IEEE Sens. J. 2015, 15, 4086-4092. [CrossRef]

9. Wang, G.; Liu, J.; Zheng, Z.; Yi, Y.; Xiao, J.; Bian, Y. Suspended core fiber integrated microfluidic chip for surface plasmon resonance enhanced biosensing. In Proceedings of the 2011 IEEE Photonics Conference (PHO), Arlington, VA, USA, 9-13 October 2011.

10. Kashyap, R.; Nemova, G. Surface Plasmon Resonance-Based Fiber and Planar Waveguide Sensors. J. Sens. 2009, 2009, 645162. [CrossRef]

11. Wang, G.; Liu, J.; Zheng, Z.; Xiao, J.; Zhang, J. A fast response tilted fiber Bragg grating fluid refractometer using an exposed-hole microstructured optical fiber. In Proceedings of the 21st International Conference on Optical Fiber Sensors, Ottawa, ON, Canada, 15-19 May 2011.

12. Han, Y.; Oo, M.K.; Sukhishvili, S.; Du, H. Photonic Crystal Fiber as an Optofluidic Platform for Surface-Enhanced Raman Scattering. In Proceedings of the 2nd Workshop on Specialty Optical Fibers and Their Applications (WSOF-2), Oaxaca, Mexico, 13-15 October 2010.

13. Liu, Y.; Xu, S.; Tang, B.; Wang, Y.; Zhou, J.; Zheng, X.; Zhao, B.; Xu, W. Note: Simultaneous measurement of surface plasmon resonance and surface-enhanced Raman scattering. Rev. Sci. Instrum. 2010, 81, 036105. [CrossRef] [PubMed] 
14. Liscidini, M.; Galli, M.; Patrini, M.; Dacarro, G.; Bajoni, D.; Loo, R.W.; Goh, M.C.; Shi, M.; Sipe, J.E. Enhancement of light-matter interaction using surface states in photonic crystal structures. In Proceedings of the Quantum Sensing and Nanophotonic Devices VII, San Francisco, CA, USA, 23-28 January 2010.

15. Hautakorpi, M.; Mattinen, M.; Ludvigsen, H. Surface-plasmon-resonance sensor based on three-hole microstructured optical fiber. Opt. Express 2008, 16, 8427-8432. [CrossRef] [PubMed]

16. Guan, C.; Yuan, L. Surface plasmon resonance refractive index fibersensor with holeassisted structure. In Proceedings of the 21st International Conference on Optical Fiber Sensors, Ottawa, ON, Canada, 15-19 May 2011.

17. Weber, M.J. Hand Book of Optical Materials; CRC Press LLC: Boca Raton, FL, USA, 2003.

18. Johnson, P.B.; Christy, R.W. Optical constants of the noble metals. Phys. Rev. B 1972, 6, 4370-4379. [CrossRef]

19. Hassani, A.; Skorobogatiy, M. Design criteria for microstructured-optical-fiber-based surface-plasmonresonance sensors. J. Opt. Soc. Am. 2007, 24, 1423-1429. [CrossRef]

20. Hassani, A.; Skorobogatiy, M. Design of the microstructured optical fiber-based surface plasmon resonance sensors with enhanced microfluidics. Opt. Express 2006, 14, 11616-11621. [CrossRef] [PubMed]

21. Azio, P.J.; Amezcua-Correa, A.; Finlayson, C.E.; Hayes, J.R.; Scheidemantel, T.J.; Baril, N.F.; Jackson, B.R.; Won, D.J.; Zhang, F.; Margine, E.R.; et al. Microstructured optical fibers as high-pressure micro fluidic reactors. Science 2006, 311, 1583-1586.

22. Harrington, J.A. A review of IR transmitting, hollow waveguides. Fiber Integr. Opt. 2000, 19, $211-227$. [CrossRef]

23. Lipomi, D.J.; Martinez, R.V.; Kats, M.A.; Kang, S.H.; Kim, P.; Aizenberg, J.; Capasso, F.; Whitesides, G.M. Patterning the Tips of Optical Fibers with Metallic Nanostructures Using Nanoskiving. Nano Lett. 2011, 11, 632-636. [CrossRef] [PubMed]

(C) 2018 by the authors. Licensee MDPI, Basel, Switzerland. This article is an open access article distributed under the terms and conditions of the Creative Commons Attribution (CC BY) license (http:/ / creativecommons.org/licenses/by/4.0/). 